\title{
Anterior lumbar discectomy and fusion for acute cauda equina syndrome caused by recurrent disc prolapse: report of 3 cases
}

\author{
Kimberly-Anne Tan, MBBS, ${ }^{1,2}$ Mathew D. Sewell, FRCS, ${ }^{1}$ Yma Markmann, MD, ${ }^{1}$ \\ Andrew J. Clarke, FRCS, ${ }^{1}$ Oliver M. Stokes, FRCS, ${ }^{1}$ and Daniel Chan, FRCS ${ }^{1}$ \\ ${ }^{1}$ Exeter Spine Unit, Princess Elizabeth Orthopaedic Centre, Royal Devon and Exeter NHS Foundation Trust, Exeter, United \\ Kingdom; and 'University of New South Wales, Faculty of Medicine, Sydney, New South Wales, Australia
}

\begin{abstract}
There is a lack of information and consensus regarding the optimal treatment for recurrent disc herniation previously treated by posterior discectomy, and no reports have described an anterior approach for recurrent disc herniation causing cauda equina syndrome (CES). Revision posterior decompression, irrespective of the presence of CES, has been reported to be associated with significantly higher rates of dural tears, hematomas, and iatrogenic nerve root damage. The authors describe treatment and outcomes in 3 consecutive cases of patients who underwent anterior lumbar discectomy and fusion (ALDF) for CES caused by recurrent disc herniations that had been previously treated with posterior discectomy. All 3 patients were operated on within 12 hours of presentation and were treated with an anterior retroperitoneal lumbar approach. Follow-up ranged from 12 to 24 months. Complete retrieval of herniated disc material was achieved without encountering significant epidural scar tissue in all 3 cases. No perioperative infection or neurological injury occurred, and all 3 patients had neurological recovery with restoration of bladder and bowel function and improvement in back and leg pain. ALDF is one option to treat CES caused by recurrent lumbar disc prolapse previously treated with posterior discectomy. The main advantage is that it avoids dissection around epidural scar tissue, but the procedure is associated with other risks and further evaluation of its safety in larger series is required.
\end{abstract}

https://thejns.org/doi/abs/10.3171/2017.1.SPINE16352

KEY WORDS anterior lumbar discectomy and fusion; cauda equina syndrome; recurrent disc herniation

$\mathrm{P}$ OSTERIOR decompression via total laminectomy and discectomy is the most commonly performed surgical procedure for the treatment of acute cauda equina syndrome (CES) caused by a canal-filling disc. There is no consensus and a lack of information to advise on the management of acute CES caused by a recurrent disc prolapse as the condition is rare. Irrespective of whether CES is present, the practice for many surgeons is posterior discectomy for the primary disc herniation, revision posterior discectomy for a recurrence, and a subsequent posterior discectomy and fusion if a second recurrence occurs. However, the addition of fusion is controversial and not always necessary.
Revision posterior decompression is associated with a significantly higher rate of dural tear, hematoma, and iatrogenic nerve root damage due to the presence of epidural fibrosis. ${ }^{11}$ An anterior discectomy, in theory, would lessen these potential risks because it would avoid the epidural scar tissue within the canal; however, it would present the surgeon with a different set of risks, particularly visceral and vascular injury.

The rationale for fusion in the revision discectomy setting is to treat postdiscectomy back pain, address segmental instability (which may be caused by extensive posterior bone resection required for safe access to the disc), and enable complete disc removal to eliminate the risk of future

ABBREVIATIONS ALDF = anterior lumbar discectomy and fusion; $C E S$ = cauda equina syndrome; $P L L$ = posterior longitudinal ligament.

ACCOMPANYING EDITORIAL See pp 349-351. DOI: 10.3171/2017.2.SPINE1721.

SUBMITTED March 25, 2016. ACCEPTED January 5, 2017.

INCLUDE WHEN CITING Published online July 14, 2017; DOI: 10.3171/2017.1.SPINE16352. 
recurrence. In the presence of a second disc herniation, particularly a large one as in CES, fusion may also prevent sequelae of removal of large fragments of disc from the intervertebral space, such as loss of disc height, back pain, and deformity. Transforaminal and posterior interbody fusions have been performed in this setting with inconsistent results. ${ }^{3}$

There are no previous reports describing anterior discectomy and fusion for CES caused by recurrent lumbar disc herniation. We report on 3 cases in which anterior discectomy and fusion were performed for this condition. The rationale for anterior surgery in each of the cases described was as follows: 1) to avoid potential complications related to posterior epidural scarring, 2) to enable a more extensive discectomy to eliminate the risk of recurrence, and 3) to treat postdiscectomy back pain that had complicated the primary posterior lumbar disc surgery in each patient. All 3 patients underwent anterior lumbar discectomy and fusion (ALDF) in which a stand-alone cage was placed at the level of the recurrent pathology.

\section{Case Reports}

\section{Case 1}

A 53-year-old man presented to our institution with CES due to a recurrent L5-S1 disc prolapse. He had previously undergone 2 posterior microdiscectomies at L5S1. The patient's first surgery, 4 years previously, was a posterior L5-S1 microdiscectomy for CES caused by a canal-filling disc. Postoperatively, he recovered full bladder and bowel function and experienced complete resolution of bilateral leg pain. A recurrence of the L5-S1 disc prolapse occurred 2 years later resulting in right S-1 radicular leg pain. Revision right L5-S1 posterior discectomy successfully alleviated the leg pain, but the patient reported ongoing, intermittent low-back pain. Two years later he presented acutely with bilateral radicular leg pain, urinary incontinence, and an abnormal rectal examination with an absent bulbocavernosus reflex. MRI revealed recurrence of a large, right paracentral L5-S1 disc (Fig. 1), and a diagnosis of CES was made in view of the objective evidence of acute sacral nerve root dysfunction.

Due to concerns regarding epidural and perineural scarring following the 2 previous L5-S1 discectomies, an anterior approach for discectomy was selected. Fusion was chosen because this was the patient's third presentation for the same-level pathology and the patient had ongoing low-back pain. The patient was operated on immediately via an anterior, left-sided retroperitoneal approach to access the L5-S1 disc, which was excised under direct vision. The posterior annulus fibrosus and posterior longitudinal ligament (PLL) defects were visualized using an operating microscope, and the sequestered fragments were retrieved without difficulty through the defect. The dural tube was found to be mobile and not adherent to the sequestrated fragments. Epidural scarring was seen but not directly encountered during the decompression. A stand-alone constrained anterior cage was filled with femoral head allograft mixed with bone marrow aspirate and placed into the disc space. It was fixed with constrained locking screws to facilitate fusion through the disc space.

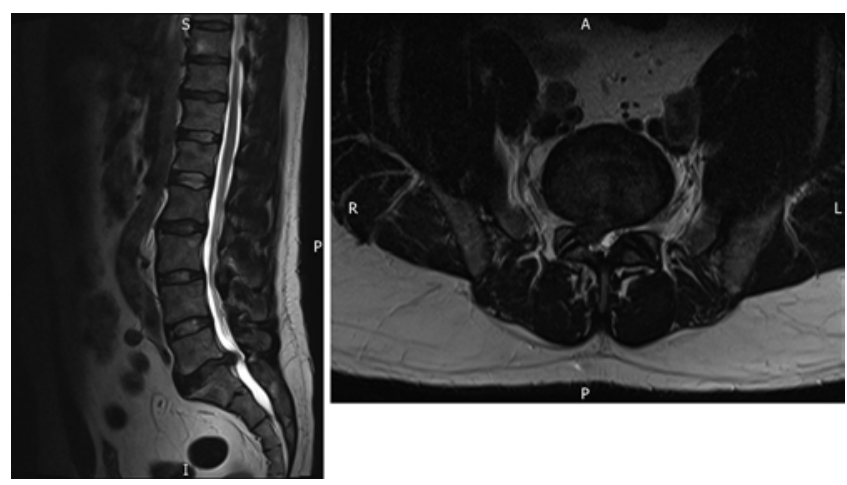

FIG. 1. Case 1. Preoperative sagittal (left) and axial (right) MR images.

On the 1st postoperative day, the patient's bilateral leg pain had resolved and the patient was able to ambulate. The patient's bulbocavernosus reflex had returned, the urinary catheter was removed, and he was able to micturate with voluntary control. Hospital discharge occurred on the 2nd postoperative day.

Postoperative MRI (Fig. 2A and B) demonstrated adequate decompression. A radiograph at 8 months demonstrated satisfactory cage positioning (Fig. $2 \mathrm{C}$ and D). At 2 years, the patient reported no significant back or leg pain and he no longer required any analgesics.

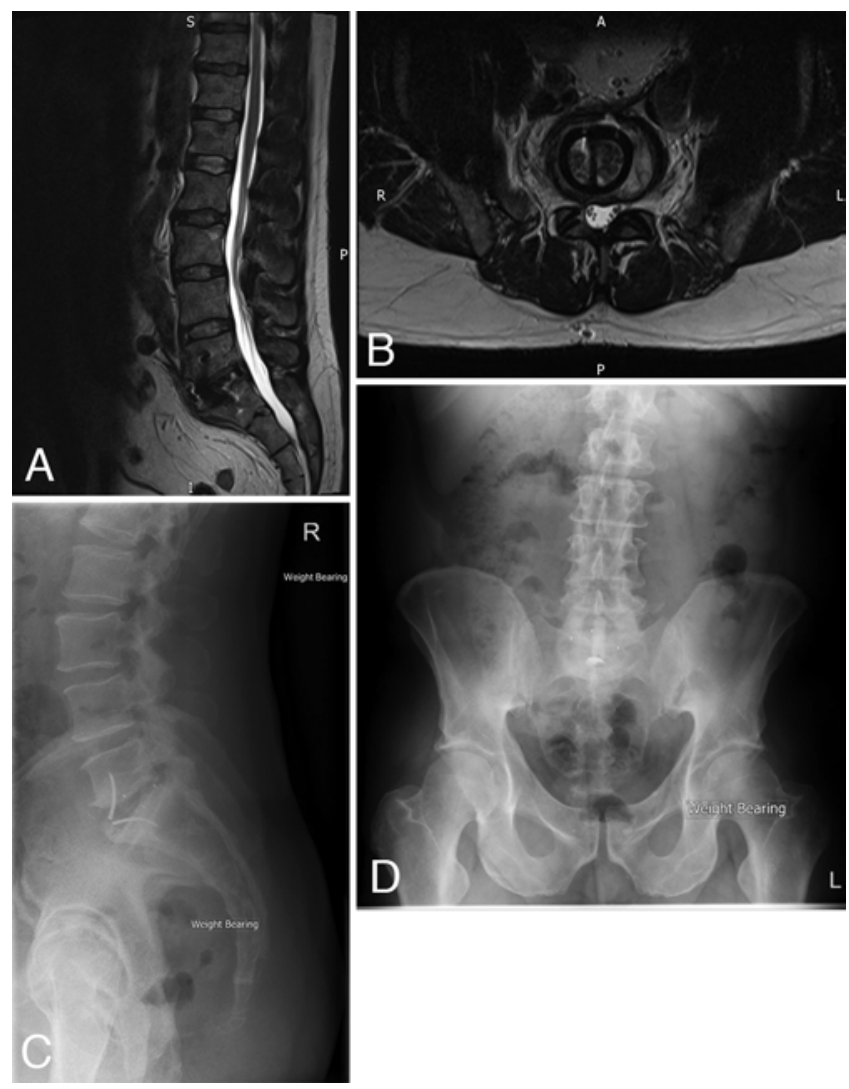

FIG. 2. Case 1. Six-week postoperative sagittal (A) and L5-S1 axial (B) $\mathrm{MR}$ images and 8-month postoperative weight-bearing lateral $(\mathbf{C})$ and anteroposterior $(\mathrm{D})$ radiographs. 


\section{Case 2}

A 39-year-old woman presented with acute onset of right leg pain and paresthesia in the S-1 nerve root distribution, perianal numbness, and painless urinary retention. Two years previously, she had undergone posterior discectomy at L5-S1 for right S-1 radicular leg pain. She reported improvement in leg pain following the surgery, but she had intermittent, residual low-back pain.

Neurological examination revealed reduced right perineal and leg sensation with no motor deficits. Her bulbocavernosus reflex was absent and her anal tone was reduced. MRI showed a large right paracentral L5-S1 disc (Fig. 3) that was causing cauda equina compression.

An ALDF was performed immediately. The anterior approach was chosen due to concerns regarding epidural scarring from the primary surgery. Intraoperatively, under the microscope, a large posterior disc fragment contiguous with the disc was found to be compressing the dural tube. The PLL was taken down to expose the dura, and the disc was removed under direct vision without the need to dissect around epidural scar tissue. The exiting L-5 and traversing S-1 nerve roots were visualized, and a scar could be seen adjacent to the S-1 nerve roots in the lateral recesses on both sides. A stand-alone anterior fusion cage was packed with allograft bone and implanted.

Immediately postoperatively, the patient's urinary symptoms improved and her bulbocavernosus reflex returned. Her leg pain improved, but leg and perianal paresthesia remained. She was discharged on Day 3 postoperatively. At her 6-week follow-up, the patient reported continued improvement in back and leg pain and she had ceased taking all neuropathic analgesics. Postoperative MRI demonstrated satisfactory cauda equina decompression (Fig. 4). At 1 year, the patient reported resolution of back and leg pain and normal bladder and bowel function. Neurological examination demonstrated reduced sensation to pinprick and light touch over the right perianal region and in the right S-1 nerve root distribution. Radiography demonstrated signs of bony fusion through the cage.

\section{Case 3}

A 30-year-old woman presented with sciatic pain in her right leg, incomplete urinary voiding, and perianal numb-

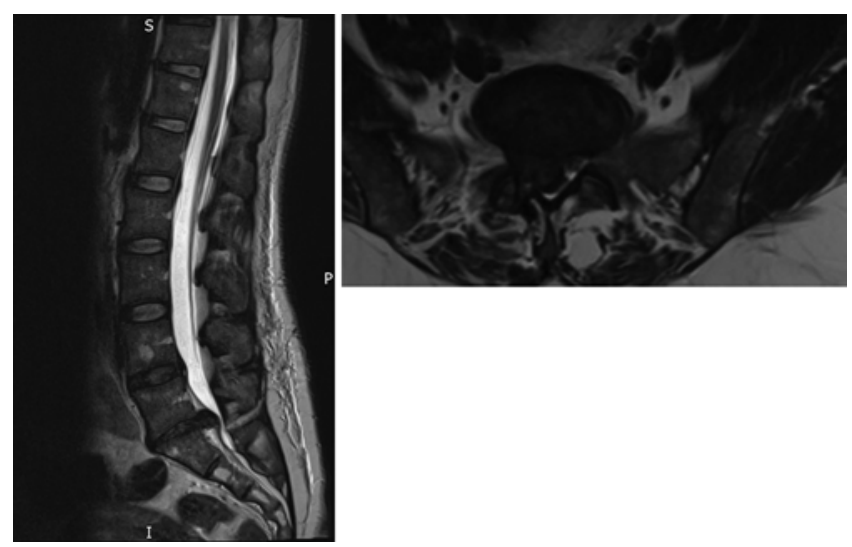

FIG. 3. Case 2. Preoperative sagittal (left) and L5-S1 axial (right) MR images.

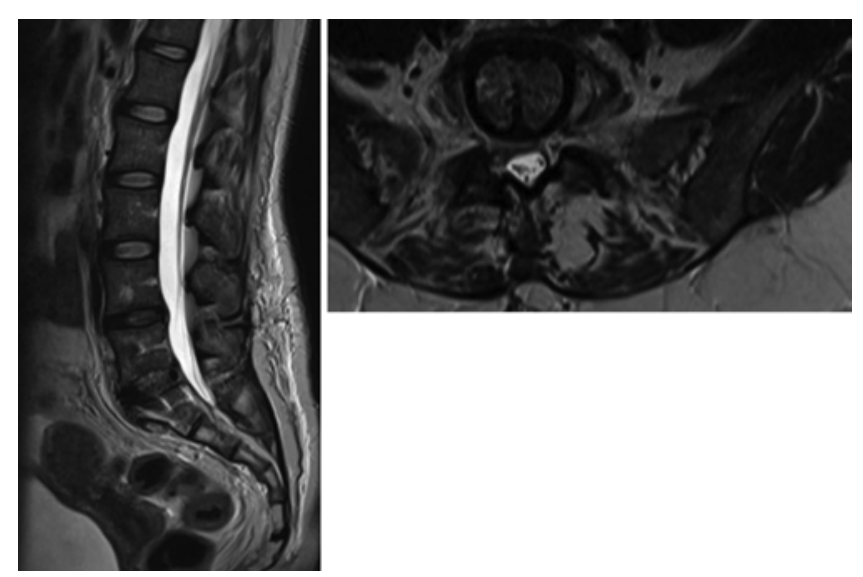

FIG. 4. Case 2. Postoperative sagittal (left) and L5-S1 axial (right) MR images showing adequate cauda equina decompression.

ness. Examination demonstrated decreased perineal sensation and reduced anal tone on rectal examination. MRI showed a large right paracentral disc at L4 -5 causing cauda equina compression (Fig. 5). Three years previously, the patient had undergone a posterior L4-5 microdiscectomy for right leg pain; the procedure had improved her leg pain but left the patient with residual low-back pain following the surgery.

A diagnosis of CES was made, and ALDF (as described in Cases 1 and 2) was performed on the same day as presentation. Intraoperatively, a defect in the posterior annulus fibrosus was identified, and loose disc fragments were seen centrally, extending distally just beyond the superior L-5 endplate, as well as into the axilla of the right L-5 nerve root. Under direct vision with the microscope, these fragments were removed. Epidural scar tissue was not encountered and a posterior lip osteophyte on L-5 was also removed.

Immediately postoperatively, the patient's right leg pain improved and her bladder and bowel function returned to normal; however, sensory dysesthesia in the leg and perianal numbness remained. Postoperative MRI demonstrat-

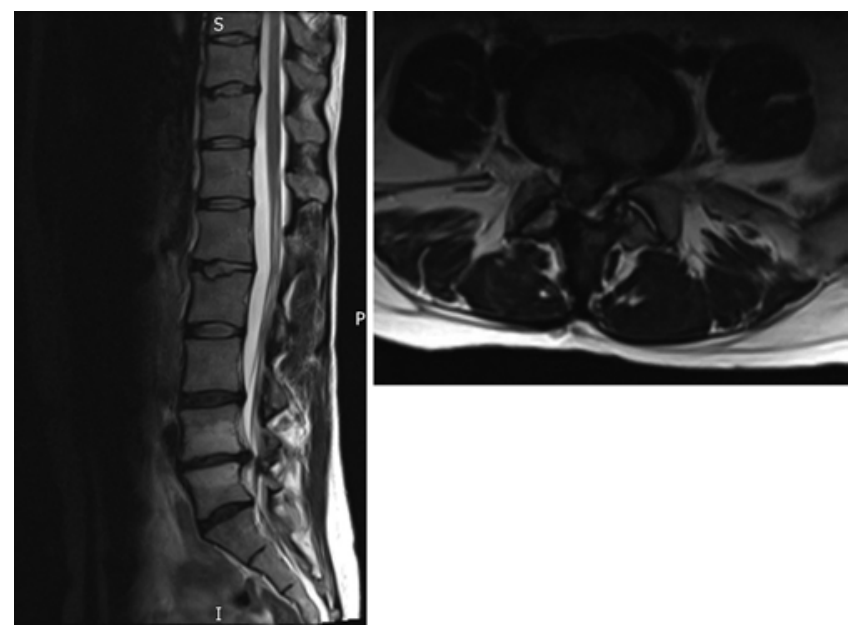

FIG. 5. Case 3. Preoperative sagittal (left) and L4-5 axial (right) MR images. 
ed adequate cauda equina decompression at L4-5 (Fig. $6 \mathrm{~A}$ and $\mathrm{B})$. At 1 year, the patient still complained of sensory dysesthesia in the right leg with persistent perianal numbness. She reported no back or leg pain, and radiography demonstrated radiographic fusion through the cage (Fig. 6C).

\section{Discussion}

The standard surgical treatment for acute disc herniation causing CES is posterior discectomy. ${ }^{2,9}$ However, in cases of recurrent disc herniation, a repeat posterior approach poses a higher risk of dural tear, especially during dissection around the nerve roots in the presence of epidural fibrosis. ${ }^{8}$ This may necessitate more extensive posterior bone removal for safe access to the disc material that needs retrieval. ${ }^{3}$ Avoidance of posterior scar tissue and treatment of residual, postdiscectomy low-back pain
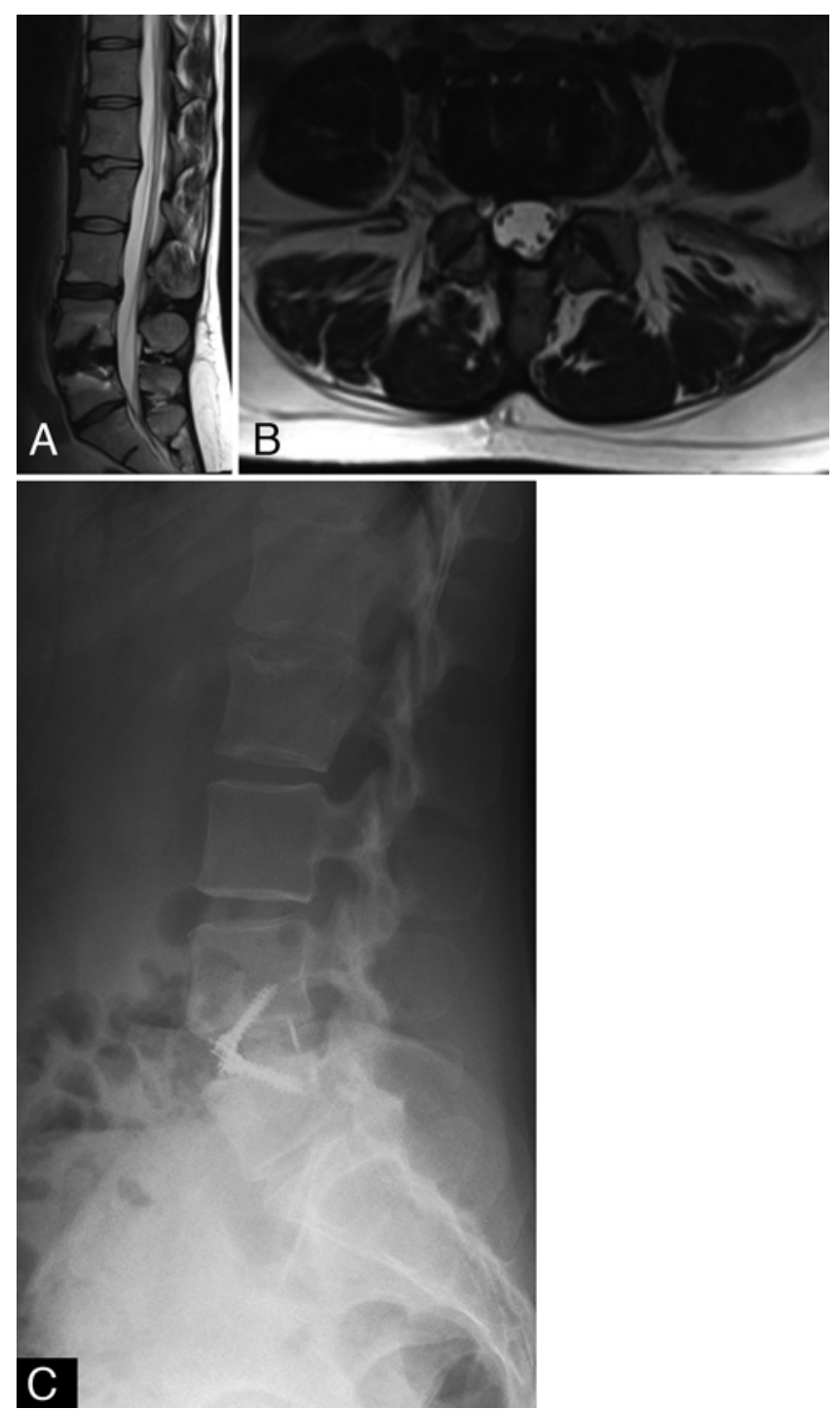

FIG. 6. Case 3. Postoperative sagittal (A) and L4-5 axial (B) MR images showing adequate cauda equina decompression. Twelve-month postoperative weight-bearing lateral radiograph $(\mathbf{C})$ showing radiographic evidence of fusion through the cage. were the rationale we used for performing primary ALDF for CES caused by recurrent disc herniation in the patients reported on in this article. The anterior approach obviated the need for dissection around epidural scar tissue, preventing the potential need for excessive posterior bone resection that might have been necessary to achieve safe access to the disc (with inherent risk of postlaminectomy instability), and addressing the residual, postdiscectomy low-back pain in each patient.

In cases in which herniated disc material is contiguous with the intervertebral disc, the anterior approach provides an added advantage because the PLL and annular tissue may act as a containing sac within which nuclear material may be more readily extracted via an anterior position. Once this material is removed, any remaining loose material within the canal, external to the herniation sac, may be extracted without the need for placing the dural tube or nerve roots under significant retraction. In our experience, the dural tube and nerve roots are placed under greater retraction when removing these intracanal disc fragments from the posterior position. In all 3 cases, we did not find it necessary to dissect around epidural scar tissue to safely remove compressive disc fragments from the canal. However, we did find that the anterior approach in this revision discectomy setting posed one additional risk. In all cases there was a thin anterior membrane over the anterior annulus of the disc, which is not usually present in a standard anterior approach to a nonoperated disc level. Presumably this was a reactive phenomenon from the previous posterior lumbar disc surgery. This may make vessel mobilization more hazardous when an anterior decompression is performed in the revision setting, which the surgeon should be aware of.

There are some disadvantages to and contraindications for the anterior approach for revision lumbar discectomy that the surgeon should be aware of including the requirement for an access surgeon, which can be difficult when the procedure is performed as an emergency procedure as in our 3 cases. In our institution, the senior surgeon carries out all the anterior approaches obviating the need for an access surgeon. This may not be the case in other centers. Additionally, vascular, ureteric, and visceral injuries are all considerable risks that are not present when using the posterior approach. We would urge caution with an anterior approach in the presence of the following factors: increasing age, great-vessel calcification, low bifurcating vasculature, history of abdominal surgery, and presence of anterior osteophytes, which can be adherent to the common iliac veins; all of these can all make the anterior approach more dangerous.

When surgery is indicated for a recurrent disc herniation, the most commonly performed revision procedure is a repeat lumbar discectomy alone without fusion. ${ }^{10}$ This is the accepted standard of care that most surgeons would follow, and it is the treatment that we advocate in our unit. However, this is a controversial topic, and there remains much debate about the optimal surgical treatment for recurrent lumbar disc herniation. Addition of a fusion to the revision discectomy has been advocated in the presence of segmental instability, chronic back pain, and CES., ${ }^{1,4,6,10}$ The rationale for fusion in CES caused by recurrent disc 
herniation is that complete disc material clearance with fusion eliminates the potential for recurrent disc herniation. Furthermore, the disc space is stabilized, removing a further potential pain source should the patient experience ongoing low-back pain. ALDF is one option to achieve fusion with acceptable long-term functional outcomes s $^{1,5}$, 7,12 and fusion rates for nonrevision discectomy patients. ${ }^{1,5}$ However outcomes in the recurrent disc herniation population have not been studied. Alternative methods of fusion, including transforaminal and posterior interbody techniques, are likely to be as equally effective. Comparison studies of techniques are difficult to perform due to the rarity of the condition.

The purpose of this case report is to describe a novel technique for the treatment for acute CES caused by recurrent lumbar disc herniation. This is a rare condition, and as such, many different treatment strategies have been proposed. When possible, we would advocate repeat posterior lumbar decompression surgery as the treatment of choice for this clinical scenario, as this procedure has a lower complication rate than a fusion procedure. If the surgeon believes fusion is appropriate because of ongoing low-back pain, the risk of instability, or the wish to remove the entire disc so that recurrence is eliminated, transforaminal and posterior lumbar interbody techniques may be appropriate and effective. These posterior procedures carry different risks and benefits than an anterior fusion procedure.

Clearly the effectiveness and safety of this novel technique need further evaluation, but from our limited experience, ALDF has been an effective treatment option in cases of recurrent disc herniation causing CES in patients who have undergone a previous posterior discectomy at the same level and who have had ongoing low-back pain as a sequela of this primary procedure. Our patients reported an improvement in their back and leg pain at 1 year and resolution of their sphincter dysfunction, and we observed no cases of infection, dural tear, or vascular or neurological injury.

\section{References}

1. Choi JY, Choi YW, Sung KH: Anterior lumbar interbody fusion in patients with a previous discectomy: minimum 2-year follow-up. J Spinal Disord Tech 18:347-352, 2005

2. Gleave JR, Macfarlane R: Cauda equina syndrome: what is the relationship between timing of surgery and outcome? $\mathbf{B r}$ J Neurosurg 16:325-328, 2002
3. Glenn JS, Yaker J, Guyer RD, Ohnmeiss DD: Anterior discectomy and total disc replacement for three patients with multiple recurrent lumbar disc herniations. Spine J 11:e1-e6, 2011

4. Greenleaf RM, Harris MB, Bono CM: The role of fusion for recurrent disk herniations. Semin Spine Surg 23:242-248, 2011

5. Inoue S, Watanabe T, Hirose A, Tanaka T, Matsui N, Saegusa $\mathrm{O}$, et al: Anterior discectomy and interbody fusion for lumbar disc herniation. A review of 350 cases. Clin Orthop Relat Res (183):22-31, 1984

6. Li Z, Tang J, Hou S, Ren D, Li L, Lu X, et al: Four-year follow-up results of transforaminal lumbar interbody fusion as revision surgery for recurrent lumbar disc herniation after conventional discectomy. J Clin Neurosci 22:331-337, 2015

7. Miyamoto K: [Long-term follow-up results of anterior discectomy and interbody fusion for lumbar disc herniation.] Nippon Seikeigeka Gakkai Zasshi 65:1179-1190, 1991 (Jpn)

8. Ozgen S, Naderi S, Ozek MM, Pamir MN: Findings and outcome of revision lumbar disc surgery. J Spinal Disord 12:287-292, 1999

9. Shapiro S: Cauda equina syndrome secondary to lumbar disc herniation. Neurosurgery 32:743-747, 1993

10. Stambough JL: An algorithmic approach to recurrent lumbar disk herniation: evaluation and management. Semin Spine Surg 20:2-13, 2008

11. Stolke D, Sollmann WP, Seifert V: Intra- and postoperative complications in lumbar disc surgery. Spine (Phila Pa 1976) 14:56-59, 1989

12. Vishteh AG, Dickman CA: Anterior lumbar microdiscectomy and interbody fusion for the treatment of recurrent disc herniation. Neurosurgery 48:334-338, 2001

\section{Disclosures}

Dr. Stokes serves as a consultant for NuVasive.

\section{Author Contributions}

Conception and design: Chan, Markmann. Acquisition of data: Chan, Tan, Markmann. Drafting the article: Tan, Markmann. Critically revising the article: Chan, Tan, Sewell, Clarke, Stokes. Reviewed submitted version of manuscript: Chan, Tan, Clarke, Stokes. Administrative/technical/material support: Chan, Tan, Sewell. Study supervision: Chan, Sewell, Stokes.

\section{Correspondence}

Daniel Chan, Exeter Spine Unit, Princess Elizabeth Orthopaedic Centre, Royal Devon and Exeter NHS Foundation Trust, Barrack Rd., Exeter EX2 5DW, United Kingdom. email: spineman.yoda@ gmail.com. 